\title{
OPEN Plasma osteopontin as a biomarker of Alzheimer's disease and vascular cognitive impairment
}

\author{
Yuek Ling Chai ${ }^{1,2}$, Joyce R. Chong ${ }^{1,2}$, Ainiah R. Raquib ${ }^{1}$, Xin $\mathrm{Xu}^{1,2}$, Saima Hilall ${ }^{1,3}$, \\ Narayanaswamy Venketasubramanian ${ }^{5}$, Boon Yeow Tan ${ }^{4}$, Alan P. Kumar ${ }^{6}$, Gautam Sethi ${ }^{1}$, \\ Christopher P. Chen ${ }^{1,2}$ \& Mitchell K. P. Lai ${ }^{1,2 \bowtie ~}$
}

Cerebrovascular disease (CeVD) and neurodegenerative dementia such as Alzheimer's disease (AD) are frequently associated comorbidities in the elderly, sharing common risk factors and pathophysiological mechanisms including neuroinflammation. Osteopontin (OPN) is an inflammatory marker found upregulated in vascular diseases as well as in AD. However, its involvement in vascular dementia (VaD) and pre-dementia stages, namely cognitive impairment no dementia (CIND), both of which fall under the spectrum of vascular cognitive impairment (VCI), has yet to be examined. Its correlations with inflammatory cytokines in cognitive impairment also await investigation. 80 subjects with no cognitive impairment $(\mathrm{NCl}), 160$ with CIND and 144 with dementia were included in a cross-sectional study on a Singapore-based memory clinic cohort. All subjects underwent comprehensive clinical, neuropsychological and brain neuroimaging assessments, together with clinical diagnoses based on established criteria. Blood samples were collected and OPN as well as inflammatory cytokines interleukin (IL)-6, IL-8 and tumor necrosis factor (TNF) were measured using immunoassays. Multivariate regression analyses showed significant associations between increased OPN and VCl groups, namely CIND with CeVD, AD with CeVD and VaD. Interestingly, higher OPN was also significantly associated with $A D$ even in the absence of CeVD. We further showed that increased OPN significantly associated with neuroimaging markers of $\mathrm{CeVD}$ and neurodegeneration, including cortical infarcts, lacunes, white matter hyperintensities and brain atrophy. OPN also correlated with elevated levels of IL-6, IL-8 and TNF. Our findings suggest that OPN may play a role in both VCI and neurodegenerative dementias. Further longitudinal analyses are needed to assess the prognostic utility of OPN in disease prediction and monitoring.

$\begin{array}{ll}\text { Abbreviations } & \\ \text { AD } & \text { Alzheimer's disease } \\ \text { ANOVA } & \text { Analyses of variance } \\ \text { APOE } & \text { Apolipoprotein E } \\ \text { ARWMC } & \text { Age-related white matter changes scale } \\ \text { AUC } & \text { Area under curve } \\ \text { CeVD } & \text { Cerebrovascular disease } \\ \text { CI } & \text { Confidence interval } \\ \text { CIND } & \text { Cognitive impairment no dementia } \\ \text { CSF } & \text { Cerebrospinal fluid } \\ \text { DSM-IV } & \text { Diagnostic and statistical manual of mental disorders, 4th edition } \\ \text { DSRB } & \text { Domain-Specific Review Board } \\ \text { EDTA } & \text { Ethylenediaminetetraacetic acid } \\ \text { FLAIR } & \text { Fluid attenuated inversion recovery }\end{array}$

${ }^{1}$ Department of Pharmacology, Yong Loo Lin School of Medicine, National University of Singapore, Unit 09-01, Centre for Translational Medicine (MD6), 14 Medical Drive, Singapore 117599, Singapore. ${ }^{2}$ Memory Aging and Cognition Centre, National University Health System, Singapore, Singapore. ${ }^{3}$ Saw Swee Hock School of Public Health, National University of Singapore, Singapore, Singapore. "St. Luke's Hospital, Singapore, Singapore. ${ }^{5}$ Raffles Neuroscience Centre, Raffles Hospital, Singapore, Singapore. ${ }^{6}$ Cancer Science Institute of Singapore and Department of Pharmacology, Yong Loo Lin School of Medicine, National University of Singapore, Singapore, Singapore. ${ }^{\varpi}$ email: Mitchell.lai@dementia-research.org 


$\begin{array}{ll}\text { IL } & \text { Interleukin } \\ \text { IQR } & \text { Interquartile range } \\ \text { MD } & \text { Mean differences } \\ \text { MFI } & \text { Median fluorescent intensity } \\ \text { MMSE } & \text { Mini-Mental State Examination } \\ \text { MoCA } & \text { Montreal Cognitive Assessment } \\ \text { MPRAGE } & \text { Magnetization prepared rapid gradient recalled echo } \\ \text { MRI } & \text { Magnetic resonance imaging } \\ \text { NCI } & \text { No cognitive impairment } \\ \text { NINCDS-ADRDA } & \text { National Institute of Neurological and Communicative Disorders and Stroke and the } \\ & \text { Alzheimer's Disease and Related Disorders Association } \\ \text { NINDS-AIRENS } & \text { National Institute of Neurological Disorders and Stroke-Association Internationale pour } \\ & \text { la Recherché et l' Enseignement en Neuroscience } \\ \text { OPN } & \text { Osteopontin } \\ \text { OR } & \text { Odds ratio } \\ \text { ROC } & \text { Receiver operating characteristic } \\ \text { RR } & \text { Risk ratios } \\ \text { SD } & \text { Standard deviation } \\ \text { SPP1 } & \text { Secreted phosphoprotein 1 } \\ \text { TNF } & \text { Tumor necrosis factor } \\ \text { VaD } & \text { Vascular dementia } \\ \text { VCI } & \text { Vascular cognitive impairment } \\ \text { WAIS-R } & \text { Wechsler Adult Intelligence Scale-revised } \\ \text { WMH } & \text { White matter hyperintensities } \\ \text { WMS-R } & \text { Wechsler Memory Scale-revised } \\ & \end{array}$

\section{Background}

Over 50 million people worldwide are affected by dementia, and the number is estimated to almost triple by the year 2050 (World Alzheimer Report 2018). While dementia is an umbrella term for symptoms associated with memory decline and deterioration in one's ability to perform activities of daily living, there are various etiologies underlying dementia, of which Alzheimer's disease (AD) and vascular dementia (VaD) are the top two commonest forms. AD is a progressive neurodegenerative disease characterized by cortical deposition of amyloid plaques and neurofibrillary tangles, whereas $\mathrm{VaD}$ is generally associated with small vessel cerebrovascular diseases (CeVD), and falls under the spectrum of vascular cognitive impairment (VCI). Nevertheless, AD and VaD are often found concomitantly as mixed dementia, and also share comorbidities and overlapping pathophysiological mechanisms. Neuroinflammation, for instance, has been observed in both AD and VaD. Studies have reported the co-localization of activated microglia and astrocytes with amyloid plaques in $\mathrm{AD}$ brains ${ }^{1}$, together with elevated levels of various pro-inflammatory cytokines in blood and cerebrospinal fluid ${ }^{2}$. Similarly, activated glial cells around vascular lesions in postmortem brains ${ }^{3,4}$ as well as increased cytokines and chemokines have been reported in blood and cerebrospinal fluid of $\mathrm{VaD}$ patients ${ }^{5-8}$. Furthermore, vascular risk factors are known to contribute to $\mathrm{AD}$ progression ${ }^{9}$. In particular, stroke, cardiovascular disease, hypertension and atherosclerosis are known risk factors of cognitive impairment and $\mathrm{AD}^{10-12}$. It has been speculated that neuroinflammation may again play a part, as deleterious inflammatory responses at the site of injury are triggered by vascular insults as well as the resultant compromise of endothelial and blood-brain barrier functions ${ }^{13,14}$. Therefore, in ongoing efforts to discover and develop blood-based biomarkers of $\mathrm{VCI}$ and $\mathrm{AD}^{15-19}$, regulators of inflammation may represent attractive targets. Of these, osteopontin (OPN) has been shown to play an important role in the pathophysiology of CeVD and inflammation.

OPN, also known as early T-lymphocyte activation or secreted phosphoprotein 1, is an extracellular phosphoprotein expressed in various tissues and cells in response to stress and injury. It may be upregulated under hypoxic conditions $^{20,21}$, atherosclerosis ${ }^{22,23}$, angioplasty ${ }^{24}$, cerebral ischemia ${ }^{25,26}$, and by pro-inflammatory cytokines such as interleukin- $1 \beta$ and interleukin- $6^{27,28}$. OPN has in turn been shown to regulate macrophage infiltration and cytokine production $^{29-31}$. In the past decade, accumulating evidence pointed to the involvement of OPN in inflammation-associated neurological disease. In $\mathrm{AD}$, higher OPN levels have been reported in the brain ${ }^{32}$ as well as in $\mathrm{CSF}^{33}$. Elevation of OPN was also observed in the plasma of patients with AD of less than 2 years, but not in patients with longer disease duration ${ }^{34}$. However, whilst studies have focused on AD, little is known about the status of OPN in VaD. The putative link with pre-dementia stages of VCI, i.e., cognitive impairment no dementia (CIND) also awaits investigation. In the present study, we aimed to examine the associations of blood OPN with AD and VCI, as well as with their neuroimaging and neurocognitive features. Given the role of OPN as a central regulator during inflammatory processes especially in regulating cytokine production, its potential associations with circulating inflammatory cytokine levels were also assessed.

\section{Methods}

Study cohort. The present case-control study consisted of subjects with CIND and dementia recruited from two study sites in Singapore (National University Hospital and Saint Luke's Hospital). Cognitively normal controls (No Cognitive Impairment, NCI), defined as cognitively normal on objective neuropsychological assessments, were recruited from both memory clinics and the community ${ }^{35}$. Institutional Review Board approval for the study, which was conducted in accordance with the Declaration of Helsinki, was obtained from 
the Singapore National Healthcare Group Domain-Specific Review Board (reference 2010/00017; study protocol DEM4233). Written informed consent was obtained for all participants or their caregivers in their preferred language prior to study recruitment.

Examination procedures. All subjects underwent standard physical, clinical, blood tests and neuropsychological assessments as well as neuroimaging scans at the National University of Singapore. Detailed study procedures have been described previously ${ }^{35}$.

Blood biomarkers measurements. Non-fasting blood was drawn from study participants into both serum-separating tubes (SST) and ethylenediaminetetraacetic acid (EDTA) tubes, and processed by centrifugation at $2000 \mathrm{~g}$ for $10 \mathrm{~min}$ at $4{ }^{\circ} \mathrm{C}$ to derive serum and plasma, respectively, which were extracted, aliquoted into Eppendorf tubes and stored at $-80^{\circ} \mathrm{C}$ until use. All blood-based biomarkers were measured in duplicates and analyzed blinded to subject characteristics and clinical status.

OPN concentrations were measured by quantitative sandwich immunoassays (Quantikine catalogue number SOST00, R\&D Systems Inc., Minneapolis, MN, USA) in accordance to manufacturer's instructions. Briefly, stored plasma samples were thawed, diluted 25-fold in calibrator diluent buffer before adding to antibody-coated plates. Stabilized chromogen tetramethylbenzidine was then added into the wells at the recommended volumes, and color development was stopped after $30 \mathrm{~min}$ by the addition of the provided stop solution. Absorbance was measured at $450 \mathrm{~nm}$ on a microplate reader (BioTek, Winooski, VT, USA) with background subtraction at $570 \mathrm{~nm}$. A standard curve ranging from 0.313 to $20 \mathrm{ng} / \mathrm{mL}$ was generated for each assay and fitted to a 4-parameter logistic model with weighted R-squared correlation coefficient $>0.99$. Sample concentrations read from the standard curve were multiplied by the dilution factor of 25 to obtain the actual OPN levels in plasma.

Inflammatory cytokines, namely IL-6, IL-8 and TNF, were measured using multiplex xMAP-based Luminex immunoassays (MILLIPLEX, catalogue number HADK2MAG-61K, Merck Millipore, Billerica, MA, USA), as described previously ${ }^{36}$. Briefly, SST serum samples were incubated overnight with a mixture of fluorescent-coded magnetic beads coated with specific capture antibody against each cytokine. This was followed by the addition of biotinylated antibody and streptavidin-phycoerythrin conjugates. The median fluorescent intensity (MFI) were measured on a Luminex 200 machine with xPONENT software. The standard curves were fitted to a 5-parameter logistic model, ranging from 0.96 to $15,000 \mathrm{pg} / \mathrm{mL}$ for IL-6, and $0.64-10,000 \mathrm{pg} / \mathrm{mL}$ for both IL-8 and TNF.

Neuropsychological assessments. Cognitive tests, which included the Mini-Mental State Examination (MMSE), the Montreal Cognitive Assessment (MoCA) and a locally validated, detailed neuropsychological test battery ${ }^{37}$, were administered to all subjects by trained research psychologists. The test battery assessed seven cognitive domains, including Executive Function, Attention, Language, Visuomotor Speed, Visuoconstruction, Visual Memory and Verbal Memory (see Additional File: Supplementary Table 1 for a summary of the component tests). Raw scores for all individual tests on the test battery were transformed to standardized z-scores using the mean values and standard deviations (SDs) of the study reference group (NCI). The z-score for each domain was then derived by averaging the $\mathrm{z}$-scores of individual tests and standardized using the means and SDs of the reference group. To obtain the global cognitive $\mathrm{z}$-score for each subject, the domain $\mathrm{z}$-scores were averaged and standardized using the means and SDs of the reference group.

Neuroimaging. Magnetic resonance imaging (MRI) scans were performed on a 3-T Siemens Magnetom Trio Tim scanner, using a 32-channel head coil, at the Clinical Imaging Research Centre, National University of Singapore. Subjects with claustrophobia, contraindications for MRI, or those who were unable to tolerate the procedure were excluded. All MRIs were graded by one radiologist and two clinicians blinded to the neuropsychological and clinical data. The sequences included T1-weighted Magnetization Prepared Rapid Gradient Recalled Echo (MPRAGE), Fluid Attenuated Inversion Recovery (FLAIR), T2-weighted and Susceptibility Weighted Imaging sequences. Presence of lacunes and cortical infarcts were defined on FLAIR and T2 sequences using STRIVE criteria $^{38}$, whereas white matter hyperintensities (WMH) were graded using the Age-Related White Matter Changes scale (ARWMC) ${ }^{39}$. Significant CeVD was defined as the presence of cortical strokes and/or $\geq 2$ lacunes and/or confluent WMH (ARWMC score $\geq 8$ ) in two regions of the brain, as described previously ${ }^{35}$.

Brain atrophy as a neuroimaging marker for neurodegeneration was also assessed on the MRI scans, with the degree of central and cortical atrophy assessed by ventricular or subarachnoid space and sulcal dilation on axial sections and rated using a 4-point scale ( 0 -normal, $1-$ mild, 2 -moderate or 3 -severe $)^{40}$. Additionally, medial temporal lobe atrophy was defined by the widening of the choroid fissure, widening of temporal horn and loss of hippocampal height as seen on coronal sections, and was graded using the 5-point Scheltens' scale (0-normal, 1 -mild, 2-mild-moderate, 3-moderate, 4 -severe $)^{41}$. The presence of significant cortical, central and medial temporal atrophy was defined by a score of $\geq 2$ on the respective scales, as previously described ${ }^{42}$.

Clinical diagnoses of cognitive impairment and dementia. Diagnoses of cognitive impairment and dementia were made at weekly consensus meetings by study clinicians and neuropsychologists. CIND was determined by clinical judgment based on published guidelines ${ }^{43}$, namely, impairment in at least one domain of the neuropsychological test battery without any significant dysfunction in activities of daily living. Participants were considered to have failed a test if they scored 1.5 standard deviation (SD) below education-adjusted cut-off values on each individual test. Failure in at least half of the tests in each domain was considered as impairment in that domain. The diagnosis of dementia was based on the diagnostic and statistical manual of mental disorders, 4th edition (DSM-IV) criteria. The etiology of dementia was further classified, with AD being diagnosed based on the National Institute of Neurological and Communicative Disorders and Stroke and the Alzheimer's 


\begin{tabular}{|c|c|c|c|c|}
\hline & $\begin{array}{l}\text { NCI } \\
(n=80)\end{array}$ & $\begin{array}{l}\text { CIND } \\
(n=158)\end{array}$ & $\begin{array}{l}\text { Dementia } \\
(\mathrm{n}=140)\end{array}$ & $p$-value \\
\hline Age, mean (SD), year & $68.8(6.3)$ & $72.0(8.2)^{*}$ & $75.7(7.8)^{* \dagger}$ & $<0.01$ \\
\hline Female, n (\%) & $41(51.2)$ & $79(50.0)$ & $86(61.4)$ & 0.11 \\
\hline Education $\leq$ elementary, n (\%) & $24(30.0)$ & $79(50.0)^{*}$ & $98(70.0)^{* \dagger}$ & $<0.01$ \\
\hline Hypertension, n (\%) & $45(56.3)$ & $108(68.4)$ & $118(84.3)^{* \dagger}$ & $<0.01$ \\
\hline Diabetes, n (\%) & $18(22.5)$ & $54(34.2)$ & $62(44.3)^{*}$ & 0.01 \\
\hline Cardiovascular disease, $\mathrm{n}(\%)$ & $4(5.0)$ & $19(12.0)$ & $26(18.6)^{*}$ & 0.01 \\
\hline Hyperlipidemia, n (\%) & $54(67.5)$ & $120(75.9)$ & $109(77.9)$ & 0.22 \\
\hline APOE $\varepsilon 4$ carrier, $\mathrm{n}(\%)$ & $14(17.5)$ & $47(29.7)$ & $45(32.1)$ & 0.06 \\
\hline Osteopontin, median (IQR), ng/ml & $59.4(22.1)$ & $65.0(28.4)$ & $81.8(42.2)^{* \dagger}$ & $<0.01$ \\
\hline
\end{tabular}

Table 1. Baseline demographic characteristics of control and cases $(n=378)$. Bold values indicates $p<0.05$ using ANOVA or Chi-square tests. CIND, cognitive impairment no dementia; IQR, interquartile range; $\mathrm{n}$, number; NCI, non-cognitive impairment; SD, standard deviation. ${ }^{*}$ Significantly different from NCI (post-hoc test, $p<0.05) .{ }^{\dagger}$ Significantly different from CIND (post-hoc test, $p<0.05$ ).

Disease and Related Disorders Association (NINCDS-ADRDA) criteria ${ }^{44}$, while vascular dementia (VaD) being diagnosed using the National Institute of Neurological Disorders and Stroke-Association Internationale pour la Recherché et l' Enseignement en Neuroscience (NINDS-AIRENS) criteria ${ }^{45}$.

Assessments of other risk factors. Risk factors such as hypertension, hyperlipidemia, diabetes and cardiovascular diseases were ascertained from clinical interview and medical records and classified as present or absent. Hypertension was defined as systolic blood pressure $\geq 140 \mathrm{mmHg}$ and/or diastolic blood pressure $\geq 90 \mathrm{mmHg}$ or use of antihypertensive medications. Diabetes mellitus was defined as glycated hemoglobin $\geq 6.5 \%$, or on medication. Hyperlipidemia was defined as total cholesterol levels $\geq 4.14 \mathrm{mM}$, or on medication. Cardiovascular disease was classified as a previous history of atrial fibrillation, congestive heart failure and myocardial infarction. Education status was categorized as low (not exceeding primary school education) or high (beyond primary school education). Apolipoprotein E (APOE) genotyping was performed as described previously ${ }^{46}$, and APOE $\varepsilon 4$ carrier status was defined as having at least one $\varepsilon 4$ allele.

Statistical analysis. Statistical analyses were performed using Statistics software (version 21, IBM SPSS, Chicago, IL, USA) and follows our previously published approach ${ }^{36,47,48}$. First, analyses of variance (ANOVA) and Chi-square tests were used to compare the characteristics of the cases and controls groups. Given that OPN was not normally distributed (Shapiro-Wilk test $p<0.001$, skewness $=2.04$, kurtosis $=5.69$ ), OPN levels were categorized into tertiles and included as a determinant, whereas CIND and dementia were defined as outcomes. Binary logistic regression analysis with odds ratios (OR) and 95\% confidence intervals (CI) were first computed for CIND and AD. Further regression analyses were performed for both CIND and AD stratified by significant $\mathrm{CeVD}$ defined by MRI. The models were adjusted for age, education, APOE $\varepsilon 4$ carrier, hypertension, diabetes and heart disease as covariates, as these variables were not matched between groups (see Table 1).

Diagnostic accuracies of OPN were assessed with logistic regression models and receiver operating characteristic (ROC) curve analyses, using $\mathrm{R}$ statistical software ${ }^{49}$ with the $p R O C$ package $^{50}$. Predicted probabilities of the continuous plasma OPN values in discriminating each diagnostic class (i.e. CIND without CeVD, CIND with $\mathrm{CeVD}, \mathrm{AD}$ without $\mathrm{CeVD}, \mathrm{AD}$ with $\mathrm{CeVD}$ and $\mathrm{VaD}$ ) from $\mathrm{NCI}$ or from all other diagnoses were obtained from binary logistic regression models built on the same approach described above. Predicted probabilities were then set as predictor while each diagnostic class was set of the outcome in the ROC analyses. Area under curves (AUC) and 95\% CIs of each ROC analysis were computed using DeLong's method ${ }^{51}$, whereas the sensitivity and specificity values were calculated at Youden index thresholds using bootstrap procedures with 2000 iterations s $^{52}$. Unadjusted models were first assessed, followed by adjustment to covariates listed above.

To examine the relationship between OPN and CeVD, as well as between OPN and brain atrophy, we performed multivariate regression analyses with log-transformed OPN included as a determinant and the specific brain MRI markers defined as outcomes. Poisson regression models with risk ratios (RR) and 95\% CI were constructed for the count of cortical and lacunar infarct, linear regression models with mean differences (MD) and 95\% CI were used for the ARWMC visual scores for WMH grading, and binary logistic regression with OR and 95\% CI were performed for brain atrophy. All models were first adjusted for age and gender, and subsequently with additional covariates including APOE $\varepsilon 4$ carrier, hypertension, diabetes and heart disease.

In order to explore the associations between OPN and neurocognitive performance, general linear regression analyses were performed with log-transformed OPN included as a determinant and the standardised z-scores of global as well as domain-based cognition were defined as outcomes, adjusting for age, gender, education, hypertension, diabetes and heart disease. Similarly, to examine the associations between OPN and other circulating inflammatory markers (i.e. IL-6, IL-8 and TNF), general linear regression analyses were performed with logtransformed OPN included as a determinant and the individual cytokines defined as outcomes, adjusting for age, hypertension, hyperlipidemia, diabetes and heart disease. $P$ values $<0.05$ were considered statistically significant. 


\begin{tabular}{|l|l|l|}
\hline Osteopontin & $\begin{array}{l}\text { CIND }(\mathbf{n}=\mathbf{1 5 8}) \\
\text { OR }(\mathbf{9 5} \% \mathbf{C I})^{\mathbf{a}}\end{array}$ & $\begin{array}{l}\text { Dementia }(\mathbf{n}=\mathbf{1 4 0}) \\
\text { OR }(\mathbf{9 5 \%} \mathbf{C I})^{\mathbf{a}}\end{array}$ \\
\hline 1st tertile & 1 & 1 \\
\hline 2nd tertile & $0.8(0.4-1.6)$ & $1.5(0.7-3.5)$ \\
\hline 3rd tertile & $2.1(0.9-4.8)$ & $\mathbf{8 . 7}(\mathbf{3 . 3}-23.1)$ \\
\hline
\end{tabular}

Table 2. Association between osteopontin and cognitive impairment, expressed in odds ratios (OR) and $95 \%$ confidence interval (CI). Bold values indicates $p<0.05$ using binary logistic regression. CIND, cognitive impairment no dementia; n, number; OR, odds ratio; CI, confidence interval. a Adjusted for age, education, hypertension, diabetes and cardiovascular disease.

\begin{tabular}{|c|c|c|c|c|c|}
\hline \multirow[b]{3}{*}{ Osteopontin } & \multicolumn{5}{|c|}{ Significant cerebrovascular disease (CeVD) } \\
\hline & \multicolumn{2}{|c|}{ Absence } & \multicolumn{3}{|l|}{ Presence } \\
\hline & $\begin{array}{l}\text { CIND without CeVD } \\
(\mathbf{n}=79) \\
\text { OR }(95 \% \mathrm{CI})^{\mathrm{a}}\end{array}$ & $\begin{array}{l}\text { AD without CeVD } \\
(n=40) \\
\text { OR }(95 \% \mathrm{CI})^{\mathrm{a}}\end{array}$ & $\begin{array}{l}\text { CIND with CeVD } \\
(\mathbf{n}=79) \\
\text { OR }(95 \% \mathrm{CI})^{\mathrm{a}}\end{array}$ & $\begin{array}{l}\text { AD with CeVD } \\
(n=69) \\
\text { OR }(95 \% \mathrm{CI})^{\mathrm{a}}\end{array}$ & $\begin{array}{l}\text { VaD } \\
(n=31) \\
\text { OR }(95 \% \mathrm{CI})^{\mathrm{a}, \mathrm{b}}\end{array}$ \\
\hline 1st tertile & 1 & 1 & 1 & 1 & 1 \\
\hline 2nd tertile & $0.5(0.2-1.0)$ & $1.1(0.2-5.9)$ & $1.3(0.6-3.0)$ & $1.2(0.4-3.6)$ & $3.3(0.8-41.7)$ \\
\hline 3rd tertile & $1.5(0.6-3.9)$ & $15.3(3.2-73.6)$ & $3.1(1.1-8.5)$ & $5.1(1.5-16.6)$ & $8.6(1.8-41.7)$ \\
\hline
\end{tabular}

Table 3. Association between osteopontin and cognitive impairment stratified by presence of significant cerebrovascular disease (CeVD), expressed in odds ratios (OR) and 95\% confidence interval (CI). Bold values indicates $p<0.05$ using binary logistic regression. Abbreviations: CeVD, cerebrovascular disease; CIND, cognitive impairment no dementia; $\mathrm{AD}$, Alzheimer's disease; VaD, vascular dementia; OR, odds ratio; $\mathrm{CI}$, confidence interval. ${ }^{\mathrm{A}}$ Adjusted for age, education, hypertension, diabetes and cardiovascular disease. ${ }^{b}$ Estimated using Firth logistic regression due to separation where hypertension perfectly predicts VaD.

Ethics approval and consent to participate. Institutional Review Board approval for the study was obtained from the Singapore National Healthcare Group Domain-Specific Review Board (reference 2010/00017; study protocol DEM4233). Written informed consent was obtained for all participants in their preferred language prior to study recruitment.

Consent for publication. All authors gave consent for publication.

\section{Results}

Baseline characteristics of study subjects. A total of 459 subjects were recruited from August 2010 to July 2015, of which 378 had sufficient MRI data and baseline plasma available for OPN analysis. In this study, there were 80 with (21.2\%) NCI, 158 (41.8\%) with CIND, and 140 (37.0\%) with dementia, of whom $109(28.8 \%)$ were $\mathrm{AD}$ while $31(8.2 \%)$ were $\mathrm{VaD}$. Table 1 shows the baseline demographic characteristics and plasma OPN levels of the study cohort. Cases were significantly older, had lower education and higher prevalence of hypertension, diabetes and cardiovascular disease compared to NCI.

Associations of OPN with CIND and dementia in the presence or absence of significant CeVD. Table 1 shows that plasma OPN levels were significantly higher in dementia compared to both NCI and CIND (Kruskal-Wallis with Dunn's post-hoc test, $\mathrm{p}<0.001$ ). After adjustment for risk factors, significant association was only observed between higher OPN and dementia ( $\mathrm{OR}=8.7 ; 95 \% \mathrm{CI} 3.3$ to 23.1 ), but not with CIND (OR=2.1; 95\% CI 0.9 to 4.8), as shown in Table 2. Given the possible effect of CeVD, logistic regression analyses were repeated after segregating the cognitive groups based on the subjects' CeVD status. As seen in Table 3, higher OPN levels were significantly associated with all VCI groups, namely CIND with CeVD ( $\mathrm{OR}=3.1 ; 95 \% \mathrm{CI} 1.1$ to 8.5$), \mathrm{AD}$ with $\mathrm{CeVD}(\mathrm{OR}=5.1 ; 95 \% \mathrm{CI} 1.5$ to 16.6$)$ and $\mathrm{VaD}(\mathrm{OR}=8.6 ; 95 \% \mathrm{CI} 1.8$ to 41.7). Interestingly, in the absence of significant CeVD, higher OPN levels were also significantly associated with $\mathrm{AD}(\mathrm{OR}=15.3 ; 95 \% \mathrm{CI} 3.2$ to 73.6$)$, but not with CIND without $\mathrm{CeVD}(\mathrm{OR}=1.5 ; 95 \% \mathrm{CI} 0.6$ to 3.9$)$.

Using ROC analyses, the potential diagnostic value of plasma OPN was assessed. Additional File: Supplementary Table 2 showed that plasma OPN are reasonably good at discriminating dementia, namely AD without $\mathrm{CeVD}(\mathrm{AUC}=0.80$, sensitivity $=72.5 \%$, specificity $=85 \%), \mathrm{AD}$ with $\mathrm{CeVD}(\mathrm{AUC}=0.71$, sensitivity $=58 \%$, specificity $=82.5 \%)$ and $\mathrm{VaD}(\mathrm{AUC}=0.73$, sensitivity $=64.5 \%$, specificity $=83.8 \%)$ from NCI controls. The diagnostic values of plasma OPN assessed by AUC, sensitivity and specificity values for each diagnostic class were markedly improved after adjusting for multiple covariates including age, education, APOE4 and other vascular risk factors (Additional File: Supplementary Table 2). Besides discriminating the cases from NCI, the potential diagnostic ability for plasma OPN in discriminating each diagnostic group from all other diagnoses was also assessed. As seen in Additional File: Supplementary Table 3, plasma OPN has potentially good diagnostic value after adjusting for multiple covariates, in particular for $\mathrm{AD}$ without $\mathrm{CeVD}(\mathrm{AUC}=0.78$, sensitivity $=92.3 \%$, specificity $=59.7 \%$ ), $\mathrm{AD}$ with $\mathrm{CeVD}(\mathrm{AUC}=0.79$, sensitivity $=81 \%$, specificity $=69.9 \%)$ and $\mathrm{VaD}(\mathrm{AUC}=0.81$, sensitivity $=95.7 \%$, specificity $=63.1 \%)$. Graphical illustrations of the ROC analyses are presented in Fig. 1. 

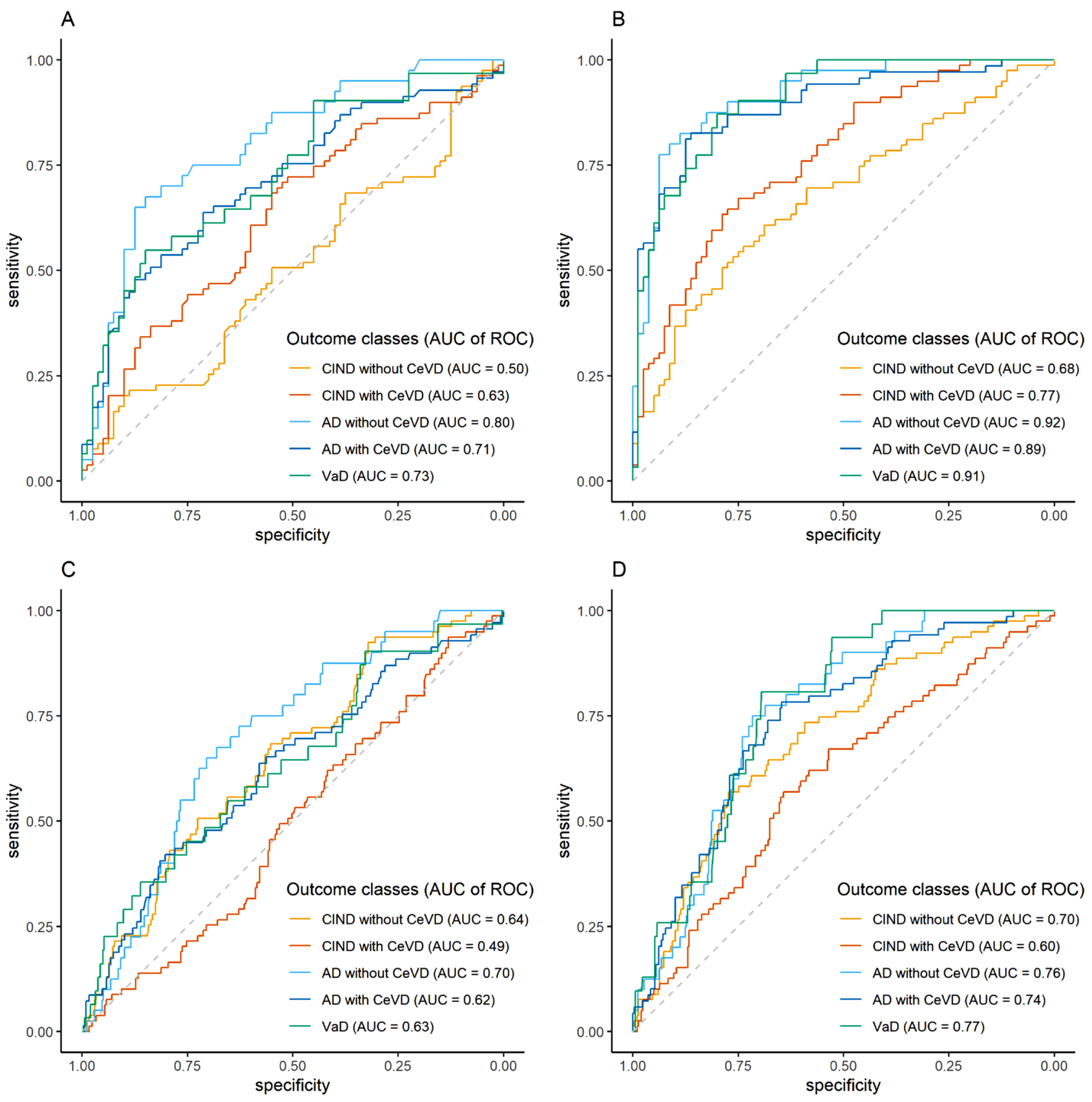

Figure 1. ROC curves of plasma OPN for discriminating each diagnostic outcome from $(\mathbf{A}, \mathbf{B}) \mathrm{NCI}$ or from $(\mathbf{C}, \mathrm{D})$ all other diagnoses $(\mathrm{n}=378)$.

Associations of OPN with MRI markers of CeVD and atrophy. The associations of OPN levels with MRI markers of CeVD and atrophy were assessed. Table 4 shows that every tenfold increase in OPN levels was significantly associated with increases in cortical infarct count ( $R R=10.72 ; 95 \% \mathrm{CI} 3.11$ to 36.93), lacune count $(\mathrm{RR}=4.27 ; 95 \% \mathrm{CI}=1.96$ to 9.28$)$ and $\mathrm{ARWMC}$ scores $(\mathrm{MD}=5.21 ; 95 \% \mathrm{CI}=2.44$ to 7.99$)$. On the other hand, Table 5 shows that every tenfold increase in OPN levels were also associated with all three neuroimaging markers of brain atrophy, namely central atrophy $(\mathrm{OR}=18.00 ; 95 \% \mathrm{CI} 3.86$ to 84.05$)$, cortical atrophy (OR $=22.67 ; 95 \%$ CI 3.65 to 140.82$)$ and medial temporal atrophy $(\mathrm{OR}=55.96$; $95 \%$ CI 9.51 to 329.32). All models were adjusted for age and gender, followed by other risk factors. All associations remain significant even when the analyses were restricted to the cognitively impaired groups (i.e. CIND and dementia) (Tables 4 and 5).

Associations of OPN with neurocognitive performance. Given that neurodegeneration and CeVD contribute to cognitive impairment, the associations between OPN and neurocognitive performance were assessed. As shown in Table 6, every tenfold increase in OPN plasma levels was significantly associated with poorer global cognition ( $\mathrm{MD}$ of $\mathrm{z}$-scores $=-5.8 ; 95 \% \mathrm{CI}-7.7$ to 3.9 ). Increased OPN was also significantly asso- 


\begin{tabular}{|c|c|c|c|}
\hline & $\begin{array}{l}\text { WMH by ARWMC scores } \\
\text { MD (95\% CI) }\end{array}$ & $\begin{array}{l}\text { Number of cortical infarcts } \\
\text { RR }(95 \% \mathrm{CI})\end{array}$ & $\begin{array}{l}\text { Number of lacunes } \\
\text { RR ( } 95 \% \text { CI) }\end{array}$ \\
\hline \multicolumn{4}{|c|}{ Osteopontin $^{\mathrm{a}}$} \\
\hline \multicolumn{4}{|c|}{ All subjects ( $\max n=378)$} \\
\hline Model I & $5.6(2.9-8.4)$ & $4.8(1.4-17.4)$ & $3.5(1.6-7.8)$ \\
\hline Model II ${ }^{\mathrm{b}}$ & $4.4(1.7-7.1)^{c}$ & $3.3(0.9-12.7)^{\mathrm{d}}$ & $1.5(0.6-3.6)^{\mathrm{e}}$ \\
\hline \multicolumn{4}{|c|}{ Cognitive impairment groups $(\max n=298)$} \\
\hline Model I ${ }^{\mathrm{b}}$ & $4.6(1.3-7.7)$ & $4.2(1.1-15.8)$ & $2.3(1.0-5.1)$ \\
\hline Model II ${ }^{\mathrm{b}}$ & $3.9(0.8-6.9)^{c}$ & $3.2(0.8-12.7)^{\mathrm{d}}$ & $1.2(0.5-2.8)^{\mathrm{e}}$ \\
\hline
\end{tabular}

Table 4. Association between osteopontin and markers of cerebrovascular disease (CeVD), expressed in risk ratios (RR) or mean differences (MD) and the respective $95 \%$ confidence interval (CI). Bold values indicates $p$ $<0.05$ using Poisson or linear regression. CeVD, cerebrovascular disease; WMH, white matter hyperintensities; ARWMC, age-related white matter changes; max n, maximum number; RR, risk ratio; $\mathrm{MD}$, mean difference; $\mathrm{CI}$, confidence interval. ${ }^{a} \mathrm{Log}$-transformed. ${ }^{\mathrm{b}}$ Adjusted for age, gender, APOE $\varepsilon 4$ carrier, hypertension, hyperlipidemia, cardiovascular disease, and additionally for ${ }^{\mathrm{c}}$ cortical infarct and lacunes, ${ }^{\mathrm{d}} \mathrm{WMH}$ and lacunes, or ${ }^{\mathrm{e}} \mathrm{WMH}$ and cortical infarct.

\begin{tabular}{|c|c|c|c|}
\hline & $\begin{array}{l}\text { Central atrophy } \\
\text { OR }(95 \% \text { CI })^{\mathrm{b}}\end{array}$ & $\begin{array}{l}\text { Cortical atrophy } \\
\text { OR }(95 \% \mathrm{CI})^{\mathrm{b}}\end{array}$ & $\begin{array}{l}\text { Medial temporal atrophy } \\
\text { OR }(95 \% \mathrm{CI})^{\mathrm{b}}\end{array}$ \\
\hline \multicolumn{4}{|l|}{ Osteopontin $^{\mathrm{a}}$} \\
\hline All subjects $(\max n=377)$ & $22.2(4.3-114.3)$ & $46.4(6.6-328.1)$ & $49.3(9.2-263.9)$ \\
\hline Cognitive impairment groups $(\max n=297)$ & $17.9(3.2-100.8)$ & $18.3(2.3-146.2)$ & $26.6(4.6-155.2)$ \\
\hline
\end{tabular}

Table 5. Association between osteopontin and brain atrophy, expressed in odds ratios (OR) and 95\% confidence interval (CI). Bold values indicates $p<0.05$ using binary logistic regression. CeVD, cerebrovascular disease; WMH, white matter hyperintensities; ARWMC, age-related white matter changes; max n, maximum number; OR, odds ratio; CI, confidence interval. ${ }^{\text {LLog-transformed. }}{ }^{\mathrm{b}}$ Adjusted for age, gender, education, hypertension, diabetes and heart disease.

\begin{tabular}{|c|c|c|}
\hline & \multicolumn{2}{|c|}{$\begin{array}{l}\text { Neurocognitive performance, } \mathrm{z} \text {-scores }(\mathrm{n}=378) \\
\text { MD }(95 \% \mathrm{CI})^{\mathrm{b}}\end{array}$} \\
\hline \multirow{8}{*}{ Osteopontin ${ }^{\mathrm{a}}$} & Global & $-5.8(-7.7,-3.9)$ \\
\hline & Executive function & $-3.5(-5.0,-2.0)$ \\
\hline & Attention & $-5.8(-8.2,-3.5)$ \\
\hline & Language & $-4.4(-6.2,-2.7)$ \\
\hline & Visuomotor speed & $-2.7(-3.7,-1.6)$ \\
\hline & Visuoconstruction & $-5.2(-6.8,-3.6)$ \\
\hline & Verbal memory & $-2.2(-3.2,-1.2)$ \\
\hline & Visual memory & $-4.5(-6.3,-2.6)$ \\
\hline
\end{tabular}

Table 6. Association of plasma osteopontin (log-transformed) with global and domain-based neurocognitive domains (in z-scores), expressed in mean difference (MD) and 95\% confidence interval (CI). Bold values indicates $\mathrm{p}<0.05$ using linear regression. MD, mean difference; CI, confidence interval. ${ }^{a}$ Log-transformed. ${ }^{\mathrm{b}}$ Adjusted for age, gender, education, hypertension, diabetes and cardiovascular disease.

ciated with worse neurocognitive performance on all individual domains, including the two memory domains namely verbal memory $(\mathrm{MD}=-2.2,95 \% \mathrm{CI}-3.2$ to -1.2$)$ and visual memory $(\mathrm{MD}=-4.5,95 \% \mathrm{CI}-6.3$ to - 2.6) as well as the five non-memory domains, namely executive function ( $\mathrm{MD}=-3.5,95 \% \mathrm{CI}-5.0$ to 2.0 ), attention $(\mathrm{MD}=-5.8,95 \% \mathrm{CI}-8.2$ to -3.5$)$, language $(\mathrm{MD}=-4.4,95 \% \mathrm{CI}-6.2$ to -2.7$)$, visuomotor speed $(\mathrm{MD}=95 \% \mathrm{CI}-2.7,-3.7$ to -1.6$)$ and visuoconstruction $(\mathrm{MD}=-5.2,95 \% \mathrm{CI}-6.8$ to -3.6$)$. All regression models were adjusted for age, gender, education and other vascular risk factors (Table 6).

Associations of OPN with inflammatory markers. General linear regression analyses were performed to examine the associations between OPN and cytokines. Out of the 378 subjects with OPN measurements available, cytokine measurement was not performed for one NCI and one AD subjects due to insufficient blood sample available, resulting in a total of 376 subjects with all four blood biomarkers analyzed. For cases whose IL-6 concentrations fell below detectable range (27 cases in NCI group, 50 cases in CIND group, 20 cases in AD group and 8 in $\mathrm{VaD}$ group), the lowest detectable value of $0.2 \mathrm{pg} / \mathrm{mL}$ was used in statistical analyses. Table 7 shows that every $1 \%$ increase in OPN was significantly associated with an increase in each of the three cytokines (in percentage), namely IL-6 $(\mathrm{MD}=0.87 ; 95 \% \mathrm{CI} 0.50$ to 1.25$)$, IL-8 $(\mathrm{MD}=0.30 ; 95 \% \mathrm{CI} 0.17$ to 0.44$)$ and TNF 


\begin{tabular}{|c|c|c|c|}
\hline & $\begin{array}{l}\text { IL-6 }^{a} \\
\text { MD }(95 \% \text { CI })^{b}\end{array}$ & $\begin{array}{l}\text { IL-8 }^{a} \\
\text { MD }(95 \% \text { CI })^{b}\end{array}$ & $\begin{array}{l}\text { TNF }^{\mathrm{a}} \\
\text { MD }(95 \% \text { CI })^{b}\end{array}$ \\
\hline \multicolumn{4}{|l|}{ Osteopontin ${ }^{a}$} \\
\hline $\begin{array}{l}\text { All subjects } \\
(\max n=376)\end{array}$ & $0.9(0.5-1.2)$ & $0.3(0.1-0.4)$ & $0.3(0.1-0.5)$ \\
\hline Cognitive impairment $(\max n=297)$ & $0.9(0.5-1.3)$ & $0.3(0.1-0.5)$ & $0.3(0.1-0.4)$ \\
\hline
\end{tabular}

Table 7. Associations between osteopontin and inflammatory markers, expressed in mean difference (MD) and $95 \%$ confidence interval (CI). Bold values indicates $p<0.05$ using linear regression. IL-6, interleukin-6; IL-8, interleukin-8; TNF, tumor necrosis factor; NCI, non-cognitive impairment; CIND, cognitive impairment no dementia; max n, maximum number; $\mathrm{MD}$, mean difference; $\mathrm{CI}$, confidence interval. ${ }^{\mathrm{a}}$ Log-transformed.

${ }^{\mathrm{b}}$ Adjusted for age, hypertension, hyperlipidemia, diabetes and cardiovascular disease.

$(\mathrm{MD}=0.31 ; 95 \% \mathrm{CI} 0.15$ to 0.48$)$. All regression models were adjusted for age and vascular risk factors, and all associations remained significant even when confined to the cognitive impairment groups only (Table 7).

\section{Discussion}

The present study found higher plasma osteopontin (OPN) in both vascular cognitive impairment as well as $\mathrm{AD}$ without significant CeVD burden. Notably, the increase in OPN levels were significantly associated with MRI markers of CeVD, namely cortical infarcts, lacunes and WMH. This is in line with a previous study which showed positive correlations between OPN and ischemic lesion volume in acute ischemic stroke patients ${ }^{53}$. Several animal stroke models also showed an increase in the mRNA levels of OPN, along with other inflammatory cytokines, suggesting that OPN may be involved in post-CeVD neuroinflammatory responses ${ }^{25,26}$. A recent study has highlighted the potential neuroprotective effect of OPN in cerebral ischemia induced inflammation and oxidative stress ${ }^{54}$, which further supports the hypothesis that the increased OPN reflects a compensatory response towards CeVD-asociated vascular damage and inflammation.

Besides CeVD, OPN may also play a role in $\mathrm{AD}$, as significant associations between higher OPN levels and $\mathrm{AD}$ in the absence of $\mathrm{CeVD}$ was observed. This is further supported by the correlation between increased OPN levels and brain atrophy observed in our study, suggesting that OPN elevation may be a response to neurodegeneration. This is in line with previous studies which implicated OPN in the clearance of pathogenic beta-amyloid $(\mathrm{A} \beta)$ proteins in $\mathrm{AD}^{55}$. Given that no significant association was observed between OPN and CIND without $\mathrm{CeVD}$, our results suggest that the compensatory response towards OPN upregulation may only be triggered in later stages of $\mathrm{AD}$ and not in pre-dementia. Furthermore, the differing trends observed between CIND with and without CeVD imply that OPN may in involved in different stages of AD vs. VCI; namely, early in VCI but later in $\mathrm{AD}$-associated neurodegeneration. Hence OPN may be a useful biomarker for identifying patients at risk of developing $\mathrm{VaD}$, as previous studies have demonstrated the likelihood of CIND patients with CeVD converting to $\mathrm{VaD}^{56,57}$. However, this requires further longitudinal study for validation.

Both neurodegeneration and $\mathrm{CeVD}$ are recognized to be the key players in cognitive impairment and dementia. We thus postulated that the atrophy- and CeVD-linked OPN is associated with clinical cognitive performance as well, and our study demonstrated for the first time that increased plasma OPN was associated with poor performance in both global as well as all domain-based cognitive functions, thus expanding upon previous findings of OPN correlations with $\mathrm{MMSE}^{33,34}$. The observed associations of OPN with all seven domainbased cognitive functions highlights the potential involvement of OPN, either directly or through regulation of neuroinflammation ${ }^{27,28}$, in the pathophysiology of cognitive impairment. This postulate is further supported by the data showing plasma OPN associations with elevation of peripheral inflammatory cytokines including IL-6, IL-8 and TNF. Our group has also previously reported associations between IL- 8 and markers of CeVD, specifically $\mathrm{WMH}^{36}$. This in turn implies that neuroinflammation may underlie the link between OPN upregulation and small vessel disease in cognitive impairment and dementia.

Our study has several limitations. Firstly, as the analysis is cross-sectional, the temporal association between OPN and the progression of cognitive impairment is not assessed. Secondly, as cases and the majority of the controls were recruited from memory clinics, who may have had a higher burden of vascular conditions or $\mathrm{CeVD}$, our findings may be less generalizable to the elderly population at large. Thirdly, different processing protocol was used for the measurement of OPN (which was measured in EDTA plasma) and other inflammatory cytokines (which were measurement in SST serum). Although blood was collected into both EDTA and SST tubes from each participant concurrently, caution needs to be taken while interpreting the data as pre-analytic blood processing procedures may affect the expression of biomarkers in the blood ${ }^{58,59}$. Lastly, the precise mechanisms underlying the involvement of OPN in VCI and AD remain unclear. The purported neuroprotective and $\mathrm{A} \beta$-clearing roles of OPN are contrasted with its function in up-regulating proinflammatory cytokines. Indeed, elevated OPN may, depending on stage and type of disease, have both beneficial and detrimental roles, the latter perhaps via maladaptive, chronic upregulation of neuroinflammation leading to tissue injury. However, more studies are needed to examine these postulated mechanisms. Nevertheless, our study's strengths include the use of 3 T-MRI to grade and classify individuals with CeVD and brain atrophy, and the use of comprehensive neuropsychological assessments to diagnose cognitive impairment and dementia as well as to examine the individual cognitive domains. Moreover, possible confounding effects of demographic characteristics and vascular risk factors were taken into account in our multivariate regression analyses. 


\section{Conclusions}

In conclusion, our findings suggest that plasma OPN is a marker for both VCI-associated CeVD as well as ADassociated brain atrophy. The current study also demonstrated OPN's positive correlation with inflammatory cytokines, as well as negative correlations with neurocognitive performance, further supporting the involvement of inflammation in VCI and AD. However, further longitudinal analyses as well as mechanistic studies are required to elucidate the precise role of OPN in disease pathogenesis and progression as well as its clinical utility as a biomarker or therapeutic target.

\section{Data availability}

The datasets used and/or analysed during the current study are available from the corresponding author on reasonable request.

Received: 13 October 2020; Accepted: 21 January 2021

Published online: 17 February 2021

\section{References}

1. Itagaki, S., McGeer, P. L., Akiyama, H., Zhu, S. \& Selkoe, D. Relationship of microglia and astrocytes to amyloid deposits of Alzheimer disease. J. Neuroimmunol. 24, 173-182. https://doi.org/10.1016/0165-5728(89)90115-X (1989).

2. Swardfager, W. et al. A meta-analysis of cytokines in Alzheimer's disease. Biol. Psychiatry 68, 930-941. https://doi.org/10.1016/j. biopsych.2010.06.012 (2010)

3. Rosenberg, G. A., Sullivan, N. \& Esiri, M. M. White matter damage is associated with matrix metalloproteinases in vascular dementia. Stroke 32, 1162-1168 (2001)

4. Olsson, B. et al. Microglial markers are elevated in the prodromal phase of Alzheimer's disease and vascular dementia. J. Alzheimer's Dis. 33, 45-53. https://doi.org/10.3233/jad-2012-120787 (2013).

5. Malaguarnera, L., Motta, M., Di Rosa, M., Anzaldi, M. \& Malaguarnera, M. Interleukin-18 and transforming growth factor-beta 1 plasma levels in Alzheimer's disease and vascular dementia. Neuropathology 26, 307-312 (2006).

6. Schmitz, M. et al. Cytokine profiles and the role of cellular prion protein in patients with vascular dementia and vascular encephalopathy. Neurobiol. Aging 36, 2597-2606. https://doi.org/10.1016/j.neurobiolaging.2015.05.013 (2015).

7. Wada-Isoe, K., Wakutani, Y., Urakami, K. \& Nakashima, K. Elevated interleukin-6 levels in cerebrospinal fluid of vascular dementia patients. Acta Neurol. Scand. 110, 124-127. https://doi.org/10.1111/j.1600-0404.2004.00286.x (2004).

8. Zuliani, G. et al. Plasma cytokines profile in older subjects with late onset Alzheimer's disease or vascular dementia. J. Psychiatr. Res. 41, 686-693. https://doi.org/10.1016/j.jpsychires.2006.02.008 (2007).

9. Snyder, H. M. et al. Vascular contributions to cognitive impairment and dementia including Alzheimer's disease. Alzheimer's Dementia J. Alzheimer's Assoc. 11, 710-717. https://doi.org/10.1016/j.jalz.2014.10.008 (2015).

10. Breteler, M. M. Vascular risk factors for Alzheimer's disease: An epidemiologic perspective. Neurobiol. Aging 21, 153-160 (2000).

11. Luchsinger, J. A. \& Mayeux, R. Cardiovascular risk factors and Alzheimer's disease. Curr. Atheroscler. Rep. 6, 261-266 (2004).

12. O'Brien, J. T. \& Markus, H. S. Vascular risk factors and Alzheimer's disease. BMC Med. 12, 218. https://doi.org/10.1186/s1291 6-014-0218-y (2014)

13. Abbott, N. J. Astrocyte-endothelial interactions and blood-brain barrier permeability. J. Anat. 200, 629-638 (2002).

14. Stanimirovic, D. \& Satoh, K. Inflammatory mediators of cerebral endothelium: A role in ischemic brain inflammation. Brain Pathol. 10, 113-126 (2000).

15. Snyder, H. M. et al. Developing novel blood-based biomarkers for Alzheimer's disease. Alzheimer's Dementia J. Alzheimer's Assoc. 10, 109-114. https://doi.org/10.1016/j.jalz.2013.10.007 (2014)

16. Zetterberg, H. Applying fluid biomarkers to Alzheimer's disease. Am. J. Physiol. Cell Physiol. 313, C3-C10. https://doi.org/10.1152/ ajpcell.00007.2017 (2017).

17. Henriksen, K. et al. The future of blood-based biomarkers for Alzheimer's disease. Alzheimer's Dementia J. Alzheimer's Assoc. 10, 115-131. https://doi.org/10.1016/j.jalz.2013.01.013 (2014).

18. Rosenberg, G. A. Vascular cognitive impairment: Biomarkers in diagnosis and molecular targets in therapy. J. Cereb. Blood Flow Metab. 36, 4-5. https://doi.org/10.1177/0271678X15609542 (2016).

19. Cipollini, V., Troili, F. \& Giubilei, F. Emerging biomarkers in vascular cognitive impairment and dementia: From pathophysiological pathways to clinical application. Int. J. Mol. Sci. https://doi.org/10.3390/ijms20112812 (2019).

20. Sodhi, C. P., Phadke, S. A., Batlle, D. \& Sahai, A. Hypoxia and high glucose cause exaggerated mesangial cell growth and collagen synthesis: Role of osteopontin. Am. J. Physiol. Renal Physiol. 280, F667-674. https://doi.org/10.1152/ajprenal.2001.280.4.F667 (2001).

21. Sodhi, C. P., Phadke, S. A., Batlle, D. \& Sahai, A. Hypoxia stimulates osteopontin expression and proliferation of cultured vascular smooth muscle cells: Potentiation by high glucose. Diabetes 50, 1482-1490 (2001).

22. Giachelli, C. M. et al. Osteopontin is elevated during neointima formation in rat arteries and is a novel component of human atherosclerotic plaques. J. Clin. Investig. 92, 1686-1696. https://doi.org/10.1172/jci116755 (1993).

23. Golledge, J., McCann, M., Mangan, S., Lam, A. \& Karan, M. Osteoprotegerin and osteopontin are expressed at high concentrations within symptomatic carotid atherosclerosis. Stroke 35, 1636-1641. https://doi.org/10.1161/01.STR.0000129790.00318.a3 (2004).

24. Wang, X. et al. Osteopontin expression in platelet-derived growth factor-stimulated vascular smooth muscle cells and carotid artery after balloon angioplasty. Arterioscler. Thromb. Vasc. Biol. 16, 1365-1372 (1996).

25. Ellison, J. A. et al. Osteopontin and its integrin receptor alpha(v)beta3 are upregulated during formation of the glial scar after focal stroke. Stroke 29, 1698-1706 (1998). (discussion 1707).

26. Hedtjarn, M., Mallard, C. \& Hagberg, H. Inflammatory gene profiling in the developing mouse brain after hypoxia-ischemia. J. Cereb. Blood Flow Metab. 24, 1333-1351. https://doi.org/10.1097/01.wcb.0000141559.17620.36 (2004).

27. Hosaka, K. et al. Monocyte chemotactic protein-1-interleukin-6-osteopontin pathway of intra-aneurysmal tissue healing. Stroke 48, 1052-1060. https://doi.org/10.1161/strokeaha.116.015590 (2017).

28. Uchibori, T. et al. IL-6 trans-signaling is another pathway to upregulate osteopontin. Cytokine 90, 88-95. https://doi.org/10.1016/j. cyto.2016.11.006 (2017).

29. Scatena, M., Liaw, L. \& Giachelli, C. M. Osteopontin: A multifunctional molecule regulating chronic inflammation and vascular disease. Arterioscler. Thromb. Vasc. Biol. 27, 2302-2309. https://doi.org/10.1161/atvbaha.107.144824 (2007).

30. Rabenstein, M. et al. Osteopontin directly modulates cytokine expression of primary microglia and increases their survival. $J$. Neuroimmunol. 299, 130-138. https://doi.org/10.1016/j.jneuroim.2016.09.009 (2016).

31. Ashkar, S. et al. Eta-1 (osteopontin): An early component of type-1 (cell-mediated) immunity. Science 287, 860-864 (2000).

32. Wung, J. K. et al. Increased expression of the remodeling- and tumorigenic-associated factor osteopontin in pyramidal neurons of the Alzheimer's disease brain. Curr. Alzheimer Res. 4, 67-72 (2007). 
33. Comi, C. et al. Osteopontin is increased in the cerebrospinal fluid of patients with Alzheimer's disease and its levels correlate with cognitive decline. J. Alzheimer's Dis. 19, 1143-1148. https://doi.org/10.3233/jad-2010-1309 (2010).

34. Sun, Y. et al. Elevated osteopontin levels in mild cognitive impairment and Alzheimer's disease. Mediators Inflamm. 2013, 615745. https://doi.org/10.1155/2013/615745 (2013).

35. Hilal, S. et al. Markers of cardiac dysfunction in cognitive impairment and dementia. Medicine 94, e297. https://doi.org/10.1097/ MD.0000000000000297 (2015).

36. Zhu, Y. et al. Serum IL-8 is a marker of white-matter hyperintensities in patients with Alzheimer's disease. Alzheimers Dement. 7, 41-47. https://doi.org/10.1016/j.dadm.2017.01.001 (2017).

37. Yeo, D. et al. Pilot validation of a customized neuropsychological battery in elderly Singaporeans. Neurol. J. South East Asia 2, 123 (1997).

38. Wardlaw, J. M. et al. Neuroimaging standards for research into small vessel disease and its contribution to ageing and neurodegeneration. Lancet Neurol. 12, 822-838. https://doi.org/10.1016/s1474-4422(13)70124-8 (2013).

39. Wahlund, L. O. et al. A new rating scale for age-related white matter changes applicable to MRI and CT. Stroke 32, 1318-1322 (2001).

40. De Leon, M. J. et al. Computed tomography evaluations of brain-behavior relationships in senile dementia of the Alzheimer's type. Neurobiol. Aging 1, 69-79. https://doi.org/10.1016/0197-4580(80)90027-5 (1980).

41. Scheltens, P., Launer, L. J., Barkhof, F., Weinstein, H. C. \& van Gool, W. A. Visual assessment of medial temporal lobe atrophy on magnetic resonance imaging: Interobserver reliability. J. Neurol. 242, 557-560. https://doi.org/10.1007/BF00868807 (1995).

42. Gyanwali, B. et al. Prevalence and association of syphilis reactivity in an Asian memory clinic population. Int. J. STD AIDS https ://doi.org/10.1177/0956462418787627 (2018).

43. Hilal, S. et al. Intracranial stenosis, cerebrovascular diseases, and cognitive impairment in Chinese. Alzheimer Dis. Assoc. Disord. 29, 12-17. https://doi.org/10.1097/WAD.0000000000000045 (2015).

44. McKhann, G. M. et al. The diagnosis of dementia due to Alzheimer's disease: Recommendations from the National Institute on Aging-Alzheimer's Association workgroups on diagnostic guidelines for Alzheimer's disease. Alzheimer's Dementia J. Alzheimer's Assoc. 7, 263-269. https://doi.org/10.1016/j.jalz.2011.03.005 (2011).

45. Román, G. C. et al. Vascular dementia: Diagnostic criteria for research studies. Report of the NINDS-AIREN International Workshop. Neurology 43, 250-260 (1993).

46. Chai, Y. L. et al. Apolipoprotein varepsilon4 is associated with dementia and cognitive impairment predominantly due to Alzheimer's disease and not with vascular cognitive impairment: A Singapore-based cohort. J. Alzheimer's Dis. 51, 1111-1118. https:// doi.org/10.3233/jad-150902 (2016).

47. Chai, Y. L. et al. Growth differentiation factor-15 and white matter hyperintensities in cognitive impairment and dementia. Medicine 95, e4566. https://doi.org/10.1097/MD.0000000000004566 (2016).

48. Chua, X. Y. et al. Immunomodulatory sphingosine-1-phosphates as plasma biomarkers of Alzheimer's disease and vascular cognitive impairment. Alzheimer's Res. Ther. 12, 122. https://doi.org/10.1186/s13195-020-00694-3 (2020).

49. R Core Team. R: A Language and Environment for Statistical Computing (R Foundation for Statistical Computing, Vienna, 2020).

50. Robin, X. et al. pROC: An open-source package for R and S+ to analyze and compare ROC curves. BMC Bioinform. 12, 77. https ://doi.org/10.1186/1471-2105-12-77 (2011).

51. DeLong, E. R., DeLong, D. M. \& Clarke-Pearson, D. L. Comparing the areas under two or more correlated receiver operating characteristic curves: A nonparametric approach. Biometrics 44, 837-845 (1988).

52. Youden, W. J. Index for rating diagnostic tests. Cancer 3, 32-35. https://doi.org/10.1002/1097-0142(1950)3:1\%3c32::aid-cncr2 820030106\%3e3.0.co;2-3 (1950).

53. Carbone, F. et al. Serum osteopontin levels are upregulated and predict disability after an ischaemic stroke. Eur. J. Clin. Investig. 45, 579-586. https://doi.org/10.1111/eci.12446 (2015).

54. Al Dera, H. Neuroprotective effect of resveratrol against late cerebral ischemia reperfusion induced oxidative stress damage involves upregulation of osteopontin and inhibition of interleukin-1beta. J. Physiol. Pharmacol. 68, 47-56 (2017).

55. Rentsendorj, A. et al. A novel role for osteopontin in macrophage-mediated amyloid-beta clearance in Alzheimer's models. Brain Behav. Immun. 67, 163-180. https://doi.org/10.1016/j.bbi.2017.08.019 (2018).

56. Meyer, J. S., Xu, G., Thornby, J., Chowdhury, M. H. \& Quach, M. Is mild cognitive impairment prodromal for vascular dementia like Alzheimer's disease?. Stroke 33, 1981-1985. https://doi.org/10.1161/01.str.0000024432.34557.10 (2002).

57. Staekenborg, S. S. et al. Progression of mild cognitive impairment to dementia: Contribution of cerebrovascular disease compared with medial temporal lobe atrophy. Stroke 40, 1269-1274. https://doi.org/10.1161/strokeaha.108.531343 (2009).

58. Nishiumi, S., Suzuki, M., Kobayashi, T. \& Yoshida, M. Differences in metabolite profiles caused by pre-analytical blood processing procedures. J. Biosci. Bioeng. https://doi.org/10.1016/j.jbiosc.2017.11.011 (2017).

59. Yu, Z. et al. Differences between human plasma and serum metabolite profiles. PLoS ONE 6, e21230. https://doi.org/10.1371/journ al.pone.0021230 (2011).

\section{Acknowledgements}

The authors would like to thank the patients and their families for their participation in this study.

\section{Author contributions}

A.P.K., G.S., C.P.C. and M.K.P.L. conceived and designed the study, as well as developed the methodology. Y.L.C., J.R.C. and A.R.R. performed the biomarker experiments. B.Y.T., S.H., X.X., N.V. and C.P.C. provided clinical data. Y.L.C., J.R.C., S.H. and X.X. analyzed the data. Y.L.C. and M.K.P.L. wrote the manuscript. C.P.C. and M.K.P.L. provided overall supervision. All authors have read, revised and approved the final version of the manuscript.

\section{Funding}

This study was supported by the National Medical Research Council (NMRC/CSA-SI/007/2016 to CPC and MKPL; NMRC/CG/M006/2017, NMRC/CG/013/2013 and NMRC/CG/NUHS/2010 to CPC).

\section{Competing interests}

The authors declare no competing interests.

\section{Additional information}

Supplementary Information The online version contains supplementary material available at https://doi. org/10.1038/s41598-021-83601-6.

Correspondence and requests for materials should be addressed to M.K.P.L. 
Reprints and permissions information is available at www.nature.com/reprints.

Publisher's note Springer Nature remains neutral with regard to jurisdictional claims in published maps and institutional affiliations.

(c) (i) Open Access This article is licensed under a Creative Commons Attribution 4.0 International License, which permits use, sharing, adaptation, distribution and reproduction in any medium or format, as long as you give appropriate credit to the original author(s) and the source, provide a link to the Creative Commons licence, and indicate if changes were made. The images or other third party material in this article are included in the article's Creative Commons licence, unless indicated otherwise in a credit line to the material. If material is not included in the article's Creative Commons licence and your intended use is not permitted by statutory regulation or exceeds the permitted use, you will need to obtain permission directly from the copyright holder. To view a copy of this licence, visit http://creativecommons.org/licenses/by/4.0/.

(C) The Author(s) 2021 